\title{
Identification of Staphylococcus aureus: DNase and Mannitol salt agar improve the efficiency of the tube coagulase test
}

\author{
David P Kateete ${ }^{1 \dagger}$, Cyrus N Kimani ${ }^{1,3 \dagger}$, Fred A Katabazi ${ }^{1}$, Alfred Okeng ${ }^{1}$, Moses S Okee ${ }^{1}$, Ann Nanteza², \\ Moses L Joloba ${ }^{1}$, Florence C Najjuka ${ }^{*}$
}

\begin{abstract}
Background: The ideal identification of Staphylococcus aureus clinical isolates requires a battery of tests and this is costly in resource limited settings. In many developing countries, the tube coagulase test is usually confirmatory for S. aureus and is routinely done using either human or sheep plasma. This study evaluated Mannitol salt agar and the deoxyribonuclease (DNase) test for improving the efficiency of the tube coagulase test in resource limited settings. The efficiency of human and sheep plasma with tube coagulase tests was also evaluated.

Methods: One hundred and eighty Gram positive, Catalase positive cocci occurring in pairs, short chains or clusters were subjected to growth on Mannitol salt agar, deoxyribonuclease and tube coagulase tests. Of these, isolates that were positive for at least two of the three tests $(n=60)$ were used to evaluate the performance of the tube coagulase test for identification of S. aureus, using PCR-amplification of the nuc gene as a gold standard.

Results: Human plasma was more sensitive than sheep plasma for the tube coagulase test (sensitivity of $91 \%$ vs. $81 \%$ respectively), but both plasmas had very low specificity (11\% and $7 \%$ respectively). The sensitivity and specificity of the tube coagulase test (human plasma) was markedly improved when Mannitol salt agar and DNase were introduced as a tri-combination test for routine identification of Staphylococcus aureus (100\% specificity and $75 \%$ sensitivity). The specificity and sensitivity of Mannitol salt agar/DNase/tube coagulase (sheep plasma) combination was $100 \%$ and $67 \%$, respectively.

Conclusion: The efficiency of the tube coagulase test can be markedly improved by sequel testing of the isolates with Mannitol salt agar, DNase and Tube coagulase. There is no single phenotypic test (including tube coagulase) that can guarantee reliable results in the identification of Staphylococcus aureus.
\end{abstract}

\section{Background}

Staphylococcus aureus is a ubiquitous commensal bacterium on human skins and anterior nares, but frequently causes severe infections in humans [1]. Rapid and direct identification of $S$. aureus is crucial for proper management of patients with skin infections, abscesses, septicemia/bacteremia, gastroenteritis, endocarditis, toxic shock syndrome and certain food intoxications $[2,3]$. In developing countries, phenotypic tests

\footnotetext{
* Correspondence: najjuka2002@yahoo.com

† Contributed equally

'Department of Medical Microbiology, School of Biomedical Sciences, Makerere University College of Health Sciences, Upper Mulago hill road Kampala, Uganda

Full list of author information is available at the end of the article
}

are the mainstay in the diagnosis of staphylococcal infections, in which coagulase tests are usually confirmatory for S. aureus [4-8]. Coagulase testing is performed using the slide coagulase (SCT) or the tube coagulase (TCT) methods [9]. Although these tests efficiently identify S. aureus, their performances vary from setting to setting and need improvement $[3,6]$.

Several laboratories in developing countries screen for presumptive Staphylococcus aureus based on growth on Mannitol salt agar (MSA) and/or DNase tests and confirmation is done with the TCT. In many settings, the use of the TCT is curtailed by reliance on human plasma, since the recommended plasmas (from rabbit, horse [10]) are either expensive or if locally available, are of poor

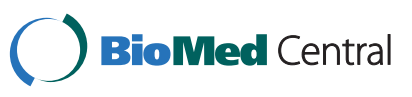


quality. Human plasma is reported to give discordant results [6]; usually obtained from blood banks as outdated materials, it contains variable amounts of CRF (Coagulase-Reacting Factor) and anti-staphylococcal antibodies [9]. This type of plasma is not recommended for coagulase tests [9]. Other factors which make human plasma inappropriate for the coagulase tests include; a high burden of viral infections (such as HIV/AIDS, Hepatitis B and $C$ ) in resource limited settings that can render the plasma risky to laboratory workers and prior to use, it must be screened for safety. There are also ethical issues accompanying the use of human specimens. Conversely, the efficiency of other plasmas in many laboratory settings varies and has to be determined for proper identification of $S$. aureus with coagulase tests.

Although coagulase tests are invaluable for identification of Staphylococcus aureus, few studies have evaluated their use in routine practice [10]. In addition, diagnostic laboratories are occasionally faced with organisms with biochemical characteristics that do not fit into the patterns of a known genus and species [11]. Furthermore, there are some problems associated with coagulase tests; firstly, some human CoNS (Coagulase Negative Staphylococci) produce clumping factor and may be falsely positive with the SCT $[9,12]$. Secondly, some staphylococci of animal origin are clumping factor negative and tube coagulase positive; these may be misidentified as $S$. aureus, unless the fermentation of Mannitol is utilized in addition [9]. Whereas Mannitol salt agar was developed for the presumptive isolation of $S$. aureus in a single step, which is convenient for diagnostic laboratories, Mannitol positive CoNS have been reported in nasal and clinical specimens from Nigeria and Japan [13,14]. Furthermore, Mannitol negative MRSA (Methicilin Resistant Staphylococcus aureus) was reported from clinical specimens in Kwazulu Natal province, South Africa [15]. In view of the above, the common identification methods for $S$. aureus were evaluated, aiming at improving the diagnosis of $S$. aureus through a combination of available phenotypic methods.

This study reveals that there is no single test (including the tube coagulase test) that can guarantee reliable results for the identification of $S$. aureus. However, improved diagnostic sensitivity and specificity of the tube coagulase test were achieved upon simultaneous testing of the isolates with DNase and Mannital salt agar. In order to improve the identification of $S$. aureus in resource limited settings, sequel testing of the isolates with Mannitol salt agar, tube DNase and coagulase is proposed.

\section{Methods}

\section{Study setting}

This study was done in the Clinical Microbiology and Molecular Biology laboratories of the Department of
Medical Microbiology, Makerere University College of Health Sciences, from January through June, 2009. It was a laboratory based study that involved frozen clinical isolates from patients' blood, cerebral spinal fluid, anterior nares, skin and wound swabs.

\section{Inclusion/exclusion criteria}

One hundred and eighty Gram positive, Catalase positive cocci occurring in pairs, short chains or clusters were subjected to growth on MSA, tube coagulase and DNase tests (see below). Of these $(\mathrm{N}=180)$, isolates that were positive for at least two of the three tests $(n=60)$ were used to evaluate the performance of the tube coagulase test for identification of $S$. aureus, using PCR-amplification of the nuc gene (which is specific for S. aureus) as the gold standard.

\section{Phenotypic identification of Staphylococcus aureus}

Isolates were incubated at $37^{\circ} \mathrm{C}$ for 24 hours on blood agar and then sub-cultured on TS (Tryptic Soya, Liofilchem, Italy) agar (Fisher, Leicestershire, UK). Single colonies were tested with tube coagulase and DNase test and growth on MSA. To evaluate the performance of the individual tests or a combination of tests, sequel testing of the isolates was done beginning with MSA, followed by DNase and TCT.

To confirm fermentation of mannitol, growth of yellow colonies on MSA (Oxoid, Cambridge, UK) surrounded by yellow zones after 24 hours of incubation at $37^{\circ} \mathrm{C}$ indicated a positive result. DNase test was performed by incubating the isolates for 24 hours at $37^{\circ} \mathrm{C}$ on DNase agar (Scharlau, Barcelona, Spain), and pouring an excess $(\sim 15 \mathrm{ml})$ of $1 \mathrm{~N} \mathrm{HCl}$. Excess acid was removed with a vacuum pipette and clear zones around the bacterial colonies indicated DNase positive colonies. For tube coagulase tests, colonies of test isolates were re-suspended in $2 \mathrm{ml}$ of citrated sheep or human plasma in sterile glass test-tubes. Since citrate is utilized by enterococci [16], pure colonies of Gram positive, Catalase positive staphylococci (catalase tests preceded coagulase reactions) were used. Positive control tubes with citrated plasma and coagulase producing strain of S. aureus ATCC 25923 (which efficiently coagulates citrated plasma) were included. To rule out citrate utilization by other microorganisms, control TCTs containing citrated plasma with Staphylococcus epidermidis ATCC 12228 were included. In addition, negative control tubes containing citrated plasma alone (with no cultures inoculated) were included. The tubes were incubated at $35^{\circ} \mathrm{C}$ for 4 hours and observed for clot formation. Where clotting did not occur, the tubes were incubated at room temperature for an additional 18 hours [9]. Tubes were studied without agitation in order not to disrupt partially formed clots. 


\section{Molecular assays}

PCR-amplification of the nuc gene was used as a baseline test. Reaction mixes were done in a Cleanspot ultraviolet workstation (COV Lab products, Michigan, USA). The reactions contained 20 pmoles each of the nuc forward and reverse primers (5'-GCGATTGATGGTGATA CGGTT-3' and 5'-AGCCAAGCCTTGACGAACTA AAGC-3', respectively, synthesized by Eurofins-MWGOperon, Ebersberg, Germany), 1.5 units of Taq DNA polymerase (Thermo-Fisher, Surry, UK) and $1 \mu$ l of custom PCR master mix (ThermoFisher, Surry, UK). In the post-amplification room, 100 ng of staphylococcal chromosomal DNA was added to the reactions as template. Amplifications were done in a Peltier thermocycler (MJ Research, Waterman, MA, USA) under the following conditions: initial denaturation at $94^{\circ} \mathrm{C}, 5 \mathrm{~min}$, followed by 37 cycles each consisting of a denaturation at $94^{\circ} \mathrm{C}$, $1 \mathrm{~min}$; primer annealing at $55^{\circ} \mathrm{C}, 0.5 \mathrm{~min}$, and extension at $72^{\circ} \mathrm{C}, 1 \mathrm{~min}$; followed by a final extension at $72^{\circ} \mathrm{C}$, 7 minutes. After this, the amplicons were mixed with $5 \mu \mathrm{l}$ of DNA loading buffer and electrophoresed in a 1\% agarose gel in TAE buffer (Tris, acetate and EDTA). Control reactions included templates of Staphylococcus aureus ATCC 25923 (positive control), Staphylococcus epidermidis ATCC 12228 and nuclease free water (negative controls).

\section{Quality control}

To minimize cross contamination, standard microbiological procedures were strictly followed. Positive and negative controls were always included in the test reactions. DNA extraction and PCR-amplifications were done in molecular laboratories that are separate from the clinical microbiology laboratory where cultures were grown. The PCR laboratory has designated sections for pre-amplification, amplification and post-amplification, with a unidirectional movement of staff.

\section{Data analysis}

The data were analyzed using a $2 \times 2$ contingency table for diagnostic specificity and sensitivity (table 1). Diagnostic sensitivities and specificities were calculated as follows:

Sensitivity $(\%)=[$ True positive $/($ True Positive + False Positive) $] \times 100$

Specificity $(\%)=[$ True Negative $/($ True Negative + False Negative) $] \times 100$

The positive predictive value $(\mathrm{PPV})(\%)=[$ True Positive/(True Positive + False Positive) $] \times 100$

The negative predictive value (NPV) $(\%)=[$ True Negative/(True Negative + False Negative) $] \times 100$.

\section{Results and discussion}

Coagulase testing is the single most reliable method for identifying Staphylococcus aureus [9]. Coagulase
Table 1 Deriving Sensitivity, Specificity, Positive/Negative Predictive Values for the common identification tests for S. aureus

\begin{tabular}{llll}
\hline $\begin{array}{l}\text { Test } \\
\text { results }\end{array}$ & $\begin{array}{l}\text { Staphylococcus } \\
\text { aureus }\end{array}$ & $\begin{array}{l}\text { Other } \\
\text { staphylococci }\end{array}$ & Total \\
\hline Positive & $\mathrm{a}$ & $\mathrm{b}$ & $\mathrm{a}+\mathrm{b}$ \\
Negative & $\mathrm{c}$ & $\mathrm{d}$ & $\mathrm{c}+\mathrm{d}$ \\
Total & $\mathrm{a}+\mathrm{c}$ & $\mathrm{b}+\mathrm{d}$ & $(\mathrm{a}+\mathrm{b})+(\mathrm{c}+\mathrm{d})$ \\
& & & $=\mathrm{n}$
\end{tabular}

Where,

$\mathrm{a}=$ True positives

$\mathrm{b}=$ False positives

$\mathrm{c}=$ False negatives

$\mathrm{d}=$ True negatives

Diagnostic sensitivity $=d /(b+d)$

Diagnostic specificity $=a /(a+c)$

Positive predictive value $(P P V)=a /(a+b)$

Negative predictive value (NPV) $=d /(d+c)$

$\mathrm{n}=60$

production can be detected using either the slide coagulase test (SCT) or the tube coagulase test (TCT). Slide coagulase detects bound coagulase (also called "clumping factor") [9], which reacts directly with fibrinogen in plasma, causing rapid cell agglutination. Negative isolates following SCT require confirmation with the superior TCT, since strains deficient in clumping factor usually produce free coagulase. Tube coagulase detects secreted extracellular free coagulase that reacts with a substance in plasma called "Coagulase-Reacting Factor" (CRF) to form a complex, which in turn reacts with fibrinogen to form fibrin (the clot) [9]. Strains of coagulase-positive-animal staphylococci have been isolated from human clinical specimens, yet some animal staphylococcal isolates also ferment mannitol [13]. This study evaluated the performance of TCTs, DNase and MSA, the phenotypic methods commonly used in the identification of Staphylococcus aureus.

\section{Detection of Staphylococcus aureus with the available phenotypic tests}

PCR-amplification of the nuc gene, which was used as a baseline test, detected 32 Staphylococcus aureus of the 60 staphylococcal isolates, see table 2. Of the 32 nucpositive Staphylococcus aureus isolates, MSA detected the highest number of isolates (30 of 32, 94\%) while the TCT (human and sheep plasma respectively) detected 29 and 27 of the 32 isolates ( $91 \%$ and $84 \%$ respectively), table 2. DNase detected the least number of isolates (24 of $32,75 \%)$.

Nine of the 32 nuc-positive Staphylococcus aureus isolates $(28 \%)$ were coagulase negative, implying that some isolates may be misidentified by the TCT as a sole test. The coagulase negative Staphylococcus aureus may probably be MRSA isolates which are reported to react weakly 
Table 2 Identification of S. aureus with the common tests in comparison to PCR-detection of the nuc gene

\begin{tabular}{|c|c|c|c|c|}
\hline \multirow[t]{2}{*}{ Other tests } & \multirow[b]{2}{*}{ Outcome } & \multicolumn{2}{|l|}{ nuc PCR } & \multirow[b]{2}{*}{ Subtotal } \\
\hline & & Positive (\% of 32 ) & Negative (\% of 28 ) & \\
\hline \multirow[t]{2}{*}{ MSA } & Positive & $30(94)$ & $6(21)$ & 36 \\
\hline & Negative & $2(6)$ & $22(79)$ & 24 \\
\hline \multirow[t]{2}{*}{ DNase } & Positive & $24(75)$ & $1(4)$ & 25 \\
\hline & Negative & $8(25)$ & $27(96)$ & 35 \\
\hline \multirow[t]{2}{*}{${ }^{1}$ Human plasma } & Positive & $29(91)$ & $25(89)$ & 54 \\
\hline & Negative & $3(9)$ & $3(11)$ & 6 \\
\hline \multirow[t]{2}{*}{ Human plasma/MSA } & Positive & $27(84)$ & $6(21)$ & 31 \\
\hline & Negative & 0 & $3(11)$ & 3 \\
\hline \multirow[t]{2}{*}{ Human plasma/DNase } & Positive & $21(60)$ & $1(4)$ & 22 \\
\hline & Negative & 0 & $3(11)$ & 3 \\
\hline \multirow[t]{2}{*}{ Human plasma/MSA/DNase } & Positive & $21(60)$ & $1(4)$ & 22 \\
\hline & Negative & 0 & $3(11)$ & 3 \\
\hline \multirow[t]{2}{*}{${ }^{2}$ Sheep plasma } & Positive & $26(81)$ & $26(93)$ & 52 \\
\hline & Negative & $6(19)$ & $2(8)$ & 8 \\
\hline \multirow[t]{2}{*}{ Sheep plasma/MSA } & Positive & $24(75)$ & $6(21)$ & 30 \\
\hline & Negative & 0 & $2(8)$ & 2 \\
\hline \multirow[t]{2}{*}{ Sheep plasma/DNase } & Positive & $18(56)$ & $1(4)$ & 19 \\
\hline & Negative & 0 & $1(4)$ & 1 \\
\hline \multirow[t]{2}{*}{ Sheep plasma/MSA/DNase } & Positive & $18(56)$ & $1(4)$ & 19 \\
\hline & Negative & 0 & $2(8)$ & 2 \\
\hline \multirow[t]{2}{*}{ MSA/DNase } & Positive & $24(74)$ & $1(4)$ & 25 \\
\hline & Negative & $2(6)$ & $22(79)$ & 24 \\
\hline
\end{tabular}

\footnotetext{
1 : tube coagulase (human plasma)

2: tube coagulase (sheep plasma)

MSA: Mannitol salt agar

DNase: Deoxyribonuclease

PCR: Polymerase Chain Reaction
}

or negatively with TCTs [17], or rare Staphylococcus aureus strains that are reported to be coagulase negative [9]. Two Staphylococcus aureus isolates (6\%) were also MSA negative. Shittu et al also reported mannitol negative Staphylococcus aureus that was methicillin resistant [15]. Furthermore, eight of the 32 nuc-positive Staphylococcus aureus isolates were DNase negative (25\%). Rao et al reported DNase negative Staphylococcus aureus but with no explanation for these findings [18]. Six staphylococci other than the nuc-confirmed Staphylococcus aureus isolates produced yellow colonies on MSA, and similar findings were reported by other investigators [19-24]. Another rare finding in this study was an isolate that was DNase positive but MSA-negative and tube coagulasenegative (i.e. non-Staphylococcus aureus). We presume this isolate could have been Staphylococcus schleiferi subsp. coagulans, which according to the National Standard Method is also DNase positive [9].

\section{Single phenotypic tests are inefficient for the} identification of Staphylococcus aureus

We used sensitivity and specificity to evaluate the performance of individual tests in detecting Staphylococcus aureus. Growth on MSA was the most sensitive (94\% sensitivity), followed by the TCT (human and sheep plasma, $91 \%$ and $81 \%$ sensitivity respectively) while the DNase test was the least sensitive (75\% sensitivity). Conversely, the DNase test was the most specific (96\% specificity) followed by MSA (79\% specificity), while the TCT (human plasma and sheep plasma) was the least specific ( $11 \%$ and $7 \%$, respectively), table 3 . Overall, of the individual tests studied, growth on MSA was the best at identifying Staphylococcus aureus (94\% sensitivity and $79 \%$ specificity). Our results slightly differ from those of Han et al, who reported sensitivity and specificity of $76.5 \%$ and $99.6 \%$, respectively, for MSA [25]. D'Souza et al, also reported sensitivity of $71 \%$, a little lower than ours [26]. The high sensitivity of MSA in detecting Staphylococcus aureus could be due to its ability to isolate pathogenic Staphylococcus aureus [27], differentiating coagulase negative staphylococci from coagulase positive staphylococci.

For tube coagulase, human plasma was more sensitive than sheep plasma ( $91 \%$ vs. $81 \%$ sensitivity), implying that using sheep plasma with TCTs may detect more false negative isolates. Thus, in this setting, it is unlikely 
Table 3 Sensitivity, Specificity, Negative/Positive Predictive values for the common diagnostic tests for clinical S. aureus

\begin{tabular}{lllll}
\hline Test & $\begin{array}{l}\text { Sensitivity } \\
(\mathbf{\%})\end{array}$ & $\begin{array}{l}\text { Specificity } \\
(\mathbf{\%})\end{array}$ & $\begin{array}{l}\text { NPV } \\
\text { (\%) }\end{array}$ & $\begin{array}{l}\text { PPV } \\
\mathbf{( \% )}\end{array}$ \\
\hline MSA & 94 & 79 & 92 & 83 \\
DNase & 75 & 96 & 77 & 96 \\
${ }^{\mathbf{}}$ Human plasma & 91 & 11 & 33 & 54 \\
${ }^{2}$ Sheep plasma & 81 & 7 & 25 & 50 \\
MSA/DNase & 96 & 92 & 92 & 96 \\
Human plasma/MSA & 33 & 100 & 100 & 82 \\
Human plasma/DNase & 75 & 100 & 100 & 95 \\
Human plasma/DNase/ & 75 & 100 & 100 & 94 \\
MSA & & & & \\
Sheep plasma/MSA & 25 & 100 & 100 & 80 \\
Sheep plasma/DNase & 50 & 100 & 100 & 95 \\
Sheep plasma/MSA & 67 & 100 & 100 & 95 \\
DNase & & & & \\
\hline
\end{tabular}

1: Tube coagulase (human plasma)

2: Tube coagulase (sheep plasma)

MSA: Mannitol salt agar

DNase: Deoxyribonuclease

PCR: Polymerase Chain Reaction

that sheep plasma will replace human plasma for routine TCTs. Our data is in agreement with previous findings in which sensitivity of $94-100 \%$ was reported by other investigators [28-30]. However, in this study, the sensitivity for human and sheep plasma was higher than what was reported in other settings, where values as low as $54.5 \%$ (human plasma) and $45.5 \%$ (sheep plasma) were reported [28]. Therefore, with coagulase tests, plasma performance varies with setting and the choice of plasma can influence the efficiency of the test. Also, the use of EDTA or citrate as anticoagulant influences the performance of the test. Noteworthy, citrate only affects coagulase reactions of enterococci where it is utilized [16], but does not affect coagulase producing organisms such as Staphylococcus aureus [16].

The DNase test had a sensitivity of $75 \%$ and a specificity of $96 \%$, which are comparable to other studies in which a sensitivity of $75 \%$ and a specificity of $100 \%$ were reported [31]. In this study, the DNase test was the most specific of all tests and had the least number of false positive isolates (there was only one false positive). This was in agreement with other reports, in which the DNase test was reported as superior to the TCT $[28,31]$. Growth on MSA was also highly specific (79\% specificity), while the least specific test was the tube coagulase (human and sheep plasma, 11\% and 7\% specificity, respectively).

In contrast, other studies reported high specificity (93.6\%) for human and sheep plasma with TCTs [32]. The low specificity in our study may partly be due to the non specific detection of other coagulase positive staphylococci, such as Staphylococcus schleiferi subspecies coagulans, Staphylococcus delphini, Staphylococcus intermedius and Staphylococcus hyicus. Staphylococcus delphini and Staphylococcus intermedius are rare clinical isolates while Staphylococcus hyicus is indeterminate (with prevalence ranging from $11 \%$ to $89 \%$ [17]). The prevalence of these isolates may be high in certain settings. Although MSA and DNase had high specificities and sensitivities, as individual tests, their use in routine identification of Staphylococcus aureus is curtailed by their ability to detect other bacterial isolates [14], and are mostly used during initial screens [6]. Indeed, Mannitol salt positive CoNS (Staphylococcus caprae, S. hemolyticus and S. saprophyticus) have been reported in Nigeria and Japan $[13,14]$. Thus, in certain settings, if used individually to identify Staphylococcus aureus, common phenotypic tests may be insufficient; some isolates will be misidentified, either as Staphylococcus aureus or CoNS.

\section{A combination of MSA and DNase improves the tube coagulase test}

We then evaluated the sensitivity and specificity of test combinations (i.e. MSA/DNase/TCT) at detecting Staphylococcus aureus, aiming at improving the performance of the TCT. We achieved absolute specificity (100\%) in detecting Staphylococcus aureus with test combinations, with the exception of the DNase/MSA combination (92\% specificity). Conversely, the sensitivity of test combinations varied, with the MSA/DNase/TCT (human plasma) being the most sensitive (75\% sensitivity), while MSA/DNase/TCT (sheep plasma) was the least sensitive (25\% sensitivity), table 3 .

A combination of MSA/DNase resulted in specificity and sensitivity of $92 \%$ and $96 \%$, respectively, and this would be the combination of choice for identification of Staphylococcus aureus. However, since both tests are not specific to Staphylococcus aureus and can detect other bacterial isolates, the dual combination can only be used to improve the TCT. Although other test combinations-MSA/DNase/TCT (human plasma) and MSA/ TCT (human plasma) had specificity of $100 \%$, they had varying sensitivity ( $75 \%$ and $33 \%$, respectively). In this line, a combination of MSA/DNase/TCT (human plasma) is better at identifying Staphylococcus aureus (100\% specificity, $75 \%$ sensitivity) than the MSA/TCT (human plasma) combination (100\% specificity, 33\% sensitivity). Likewise, the DNase/TCT (sheep plasma) and MSA/TCT (sheep plasma) and MSA/DNase/TCT (sheep plasma) combinations had an absolute specificity (100\%) but with varying sensitivity (50\%, 25\% and 67\%, respectively).

Thus, the efficiency of the tube coagulase can be improved through simultaneous testing that includes 
both DNase and MSA. For higher sensitivity and specificity, sequel identification of Staphylococcus aureus may commence with MSA, DNase and then TCT. Noteworthy, the improved specificity of the TCT did not significantly alter the initially observed high sensitivity. The use of MSA prior to TCTs/DNase is highly recommended due to the clumping factor negative and tube coagulase positive staphylococci that are increasingly being recovered from human infections. These isolates also produce a heat stable DNase and can be misidentified as Staphylococcus aureus. However, these strains can be differentiated from Staphylococcus aureus by their failure to produce acid from maltose, lactose and mannitol. Furthermore, rare strains of Staphylococcus aureus can be coagulase negative [9], yet animal isolates (S. intermidius, S. hyicus, S. delphini and S. schleiferi subsp. coagulans) can be tube coagulase positive $[9,33,34]$; differentiation of these also requires growth on MSA.

\section{Predictive values}

Growth on MSA had the highest negative predictive value (NPV) followed by DNase, while tube coagulase had the lowest NPV (which did not match the high sensitivity initially observed, see table 3 ). Conversely, test combinations gave absolute NPVs $(100 \%)$ with the exception of DNase/MSA (NPV of 92\%), table 3. The high NPVs of the test combinations, particularly those involving the TCT reveal that test combinations can be reliably used in routine identification of Staphylococcus aureus. DNase had the highest positive predictive value (PPV), followed by MSA (96\% and 83\%, respectively). Conversely, tube coagulase alone resulted in average PPVs (54\% and 50\%), see table 3. Interestingly, test combinations resulted in high PPVs, with those involving DNase having the highest PPVs (95\%), see table 3. Thus, as for NPVs, the specificity of the TCT can be improved by a simultaneous identification of the isolates with DNase and MSA.

Therefore, the ideal identification of Staphylococcus aureus clinical isolates requires a battery of tests. Staphylococcus aureus infections are more frequent than those by other bacteria, particularly in settings with high HIV/AIDS prevalence [35-37]. This warrants correct identification of the isolates to achieve better treatment outcomes. Since options for improving the sensitivity and specificity are presented, these data will improve on the identification of Staphylococcal aureus in clinical specimens.

\section{Recommendations}

For routine identification of Staphylococcus aureus from clinical specimens, we recommend simultaneous use of all the three tests (beginning with growth on MSA,
DNase and Tube coagulase) in settings where rabbit or horse plasmas are not readily available. Screening of plasmas from other species (such as rabbit, goat, pig, and bovine), which can be cheaper and safer, and reported to be more efficient than human plasma with TCTs, is recommended. In addition, genotyping of clinical staphylococcal isolates is recommended to speciate CoNS isolates, and to determine the prevalence of rare Staphylococcus aureus and animal isolates in human specimens in Uganda.

\section{Conclusions}

We have evaluated the performance of common laboratory tests used routinely in the identification of Staphylococcus aureus infections in Uganda. The identification of clinical Staphylococcus aureus still largely relies on the tube coagulase test, but it requires screening of the isolates with two additional tests (MSA and DNase) prior to TCTs, for improved efficiency. There is no single phenotypic test (including the tube coagulase test) that can guarantee reliable results in the identification of Staphylococcus aureus.

The specificity of sheep plasma was relatively low (even in combination with MSA/DNase), and may not be appropriate for the TCT in some settings. Thus, where rabbit or horse plasmas are unavailable, sheep plasma may not replace human plasma. Since human plasma is inappropriate or risky in some settings, the performance of plasmas from other animal species should be investigated for a replacement of human plasma in TCTs.

\section{Abbreviations}

ATCC: American Type Culture Collection; CoNS: Coagulase Negative Staphylococci; CRF: Coagulase Reacting Factor; DNase: Deoxyribonuclease EDTA: Ethylenediaminetetraacetic acid; HCl: Hydrochloric acid; HIV/AIDS: Human immunodeficiency virus/Acquired immunodeficiency syndrome; LB: Luria-Bertani; MRSA: Methicillin Resistant Staphylococcus aureus; MSA: Mannital salt agar; nuc: thermonuclease gene; PCR: polymerase chain reaction; PPV: Positive Predictive Value; NPV: Negative Predictive Value; STC: Slide Coagulase Test; TCT: Tube Coagulase Test; TS: Tryptic soy; TAE: Tris Acetate-EDTA

\section{Acknowledgements}

FCN and CNK thank the technical staff at the clinical microbiology laboratory (Makerere University College of Heath Sciences) for helping with microbial cultures, and Ms Geraldine Nalwadda for administrative assistance. This study obtained funding from Sida-Sarec (to FCN) through Makerere University School of Graduate Studies. DPK was supported by the Fogarty International Center training support (award \#U2RTW006879) through ICHORTA-Clinical Operational \& Health Services Research (COHRE) Training program at the Joint Clinical Research Center, Kampala, Uganda.

\section{Author details}

'Department of Medical Microbiology, School of Biomedical Sciences, Makerere University College of Health Sciences, Upper Mulago hill road, Kampala, Uganda. ${ }^{2}$ Department of Veterinary Parasitology and Microbiology, Faculty of Veterinary Medicine, Makerere University, Kampala, Uganda. ${ }^{3}$ Current Address: P.O. BOX 25085, 00603, Nairobi, Kenya. 


\section{Authors' contributions}

FCN conceived and supervised the study. DPK wrote the manuscript and cosupervised the study. CNK performed the experiments in partial fulfillment for his BSC degree. The other authors provided intellectual support to CNK and contributed to the writing of the manuscript. All authors read and approved the final manuscript.

\section{Competing interests}

The authors declare that they have no competing interests.

Received: 11 May 2010 Accepted: 13 August 2010

Published: 13 August 2010

\section{References}

1. Kluytmans J, van Belkum A, Verbrugh H: Nasal carriage of Staphylococcus aureus: epidemiology, underlying mechanisms, and associated risks. Clin Microbiol Rev 1997, 10(3):505-520.

2. Durack DT, Lukes AS, Bright DK, Duke Endocarditis S: New criteria for diagnosis of infective endocarditis: utilization of specific echocardiographic findings. Am J Med 1994, 96(3):200-209.

3. Martineau F, Picard FJ, Roy PH, Ouellette M, Bergeron MG: Species-specific and ubiquitous-DNA-based assays for rapid identification of Staphylococcus aureus. J Clin Microbiol 1998, 36(3):618-623.

4. Mugalu JNM, Kiguli S, Kaddu-Mulindwa DH: Aetiology, risk factors and immediate outcome of bacteriologically confirmed neonatal septicaemia in Mulago hospital, Uganda. Afr Health Sc 2006, 6(2):120-126.

5. Martineau F, Picard FJ, Grenier L, Roy PH, Ouellette M, Bergeron MG: Multiplex PCR assays for the detection of clinically relevant antibiotic resistance genes in staphylococci isolated from patients infected after cardiac surgery. J Antimicrob Chemother 2000, 46(4):527-534.

6. Bello CSS, Qahtani A: Pitfalls in the routine diagnosis of Staphylococcus aureus. Afr J Biotech 2006, 4(1):83-86.

7. Akpaka P, Kissoon S, Swanston W, Monteil M: Prevalence and antimicrobial susceptibility pattern of methicillin resistant Staphylococcus aureus isolates from Trinidad \& Tobago. Annal Clin Microbiol Antimicrob 2006, 5(1):16.

8. Sperber WH, Tatini SR: Interpretation of the tube coagulase test for identification of Staphylococcus aureus. Appl Microbiol 1975, 29(4):502-505.

9. Koneman EWAS, Janda WM, Schreckenberger PC, Winn WC, et al: The Gram positive cocci: Staphylococci and related organims. In Color Atlas and Textbook of Diagnostic Microbiology. Philadelphia: LippincottRavenKoneman EW , 5 1997, 551-576.

10. McDonald CL, Chapin K: Rapid identification of Staphylococcus aureus from blood culture bottles by a classic 2-hour tube coagulase test. J Clin Microbiol 1995, 33(1):50-52.

11. Woo PCY, Leung ASP, Leung KW, Yuen KY: Identification of slide coagulase positive, tube coagulase negative Staphylococcus aureus by 16S ribosomal RNA gene sequencing. Mol Path 2001, 54:244-247.

12. Freney J, Brun Y, Bes M, Meugnier H, Grimont F, Grimont PAD, Nervi C, Fleurette J: Staphylococcus lugdunensis sp. nov. and Staphylococcus schleiferi sp. nov., Two Species from Human Clinical Specimens. Int I Syst Bacteriol 1988, 38(2):168-172.

13. Kawamura Y, Hou X-G, Sultana F, Hirose K, Miyake M, Shu S-E, Ezaki T: Distribution of Staphylococcus Species among Human Clinical Specimens and Emended Description of Staphylococcus caprae. J Clin Microbiol 1998, 36(7):2038-2042.

14. Shittu A, Lin J, Morrison D, Kolawole D: Identification and molecular characterization of mannitol salt positive, coagulase-negative staphylococci from nasal samples of medical personnel and students. Med Microbiol 2006, 55(Pt 3):317-324.

15. Shittu A, Lin J, Morrison D: Molecular identification and characterization of mannitol-negative methicillin-resistant Staphylococcus aureus. Diagn Microbiol Infec Dis 2007, 57(1):93-95.

16. Bayliss BG, Hall ER: Plasma Coagulation by Organisms Other Than Staphylococcus aureus. J Bacteriol 1965, 89(1):101-105.

17. National Standard Method Coagulase SOP, UK. [http://www.hpastandardmethods.org.uk].

18. Rao JG, Qamruddin AO, Hassan IA, Burnie JP, Ganner M: Cluster of clinical isolates of epidemic methicillin-resistant Staphylococcus aureus (EMRSA) with a negative deoxyribonuclease (DNase) test-implications for laboratory diagnosis and infection control. J Hos Infec 2002, 51(3):238-239.

19. Martinez OV, Cleary T, Baker M, Civetta J: Evaluation of a mannitol-saltoxacillin-tellurite medium for the isolation of methicillin-resistant Staphylococcus aureus from contaminated sources. Diagn Microbiol Infec Dis 15(3):207-211.

20. Merlino J, Gill R, Robertson GJ: Application of lipovitellin-salt-mannitol agar for screening, isolation, and presumptive identification of Staphylococcus aureus in a teaching hospital. J Clin Microbiol 1996, 34(12):3012-3015.

21. Mir N, Sanchez M, Baquero F, Lopez B, Calderon C, Canton R: Soft SaltMannitol Agar-Cloxacillin Test: a Highly Specific Bedside Screening Test for Detection of Colonization with Methicillin-Resistant Staphylococcus aureus. J Clin Microbiol 1998, 36(4):986-989.

22. Jayaratne $P$, Rutherford $C$ : Detection of methicillin-resistant Staphylococcus aureus (MRSA) from growth on mannitol salt oxacillin agar using PCR for nosocomial surveillance. Diagn Microbiol Infec Dis 1999, 35(1):13-18.

23. Simor AE, Goodfellow J, Louie L, Louie M: Evaluation of a New Medium, Oxacillin Resistance Screening Agar Base, for the Detection of Methicillin-Resistant Staphylococcus aureus from Clinical Specimens. J Clin Microbiol 2001, 39(9):3422.

24. Zadik PM, Davies S, Whittaker S, Mason C: Evaluation of a new selective medium for methicillin-resistant Staphyloccocus aureus. J Med Microbiol 2001, 50(5):476-479.

25. Han Z, Lautenbach E, Fishman N, Nachamkin I: Evaluation of mannitol salt agar, CHROMagar Staph aureus and CHROMagar MRSA for detection of meticillin-resistant Staphylococcus aureus from nasal swab specimens. J Med Microbiol 2007, 56(1):43-46.

26. D'Souza HA, Baron EJ: BBL CHROMagar Staph aureus is Superior to Mannitol Salt for Detection of Staphylococcus aureus in Complex Mixed Infections. Am J Clin Path 2005, 123(6):806-808.

27. Blair EB, Emerson JS, Tull AH: A new medium, salt mannitol plasma agar, for the isolation of Staphylococcus aureus. Am J Clin Pathol 1967, 47(1):30-39.

28. Oranusi SG, M, Umoh VJ: Toxicity test and bacteriophage typing of Staphylococcus aureus isolates from food contact surfaces and foods prepared by families in Zaria, Nigeria. Afr J Biotech 2006, 5(4):362-365.

29. Orth DS, Chugg LR, Anderson AW: Comparison of Animal Sera for Suitability in Coagulase Testing. Appl Environ Microbiol 1971, 21(3):420-425.

30. Tager M, Hales HB: The Experimental Production of Antibodies to Staphylocoagulase. J Immunol 1948, 60(4):475-485.

31. Lagace-Wiens PRS, Alfa MJ, Manickam K, Karlowsky JA: Thermostable DNase Is Superior to Tube Coagulase for Direct Detection of Staphylococcus aureus in Positive Blood Cultures. J Clin Microbiol 2007, 45(10):3478-3479.

32. Stevens M, Geary C: Comparative evaluation of a latex test for the identification of Staphylococcus aureus. Eur J Clin Microbiol Infec Dis 1989 8(2):153-156

33. Varaldo PE, Kilpper-Balz R, Biavasco F, Satta G, Schleifer KH: Staphylococcus delphini sp. nov., a Coagulase-Positive Species Isolated from Dolphins. Int J Syst Bacteriol 1988, 38(4):436-439.

34. Vandenesch F, Lebeau C, Bes M, Lina G, Lina B, Greenland T, Benito Y, Brun Y, Fleurette J, Etienne J: Clotting activity in Staphylococcus schleiferi subspecies from human patients. J Clin Microbiol 1994, 32(2):388-392.

35. Nathoo KJ, Chigonde S, Nhembe M, Ali MH, Mason PR: Communityacquired Bacteremia in Human Immunodeficiency Virus-infected Children in Harare, Zimbabwe. Ped Infec Dis J 1996, 15(12):1092-1097.

36. Crum-Cianflone NF, Burgi AA, Hale BR: Increasing rates of communityacquired methicillin-resistant Staphylococcus aureus infections among HIV-infected persons. Int J STD AIDS 2007, 18(8):521-526.

37. Nguyen MH, Kauffman CA, Goodman RP, Squier C, Arbeit RD, Singh N, M Wagener M, Yu VL: Nasal Carriage of and Infection with Staphylococcus aureus in HIV-Infected Patients. Annal Internal Med 1999, 130(3):221-225.

doi:10.1186/1476-0711-9-23

Cite this article as: Kateete et al:: Identification of Staphylococcus aureus: DNase and Mannitol salt agar improve the efficiency of the tube coagulase test. Annals of Clinical Microbiology and Antimicrobials 2010 9:23. 